\title{
Estresse e síndrome de burnout em profissionais de odontologia
}

\author{
Stress and burnout syndrome in dental professionals
}

Síndrome de estrés y burnout en profesionales dentales

Wéllisson Luigi Lima Pinheiro ${ }^{1 *}$, Aluísio Ferreira Celestino Júnior ${ }^{2}$, Cristiano Santana Bentes Junior ${ }^{1}$, Kelly Carla Figueredo da Conceição ${ }^{1}$, Isabella Oliveira dos Santos ${ }^{2}$, Tamiris Faro Casseb², Marlene Ribeiro de Oliveira ${ }^{3}$, Caroline Oliveira Silveira ${ }^{1}$, Alessandra dos Santos Tavares Vieira ${ }^{2}$, Bárbara Geovanna Silva Souza4.

\section{RESUMO}

Objetivo: Revisar a literatura sobre as principais contribuições acerca da Síndrome de Burnout relacionadas a cirurgiões dentistas. Métodos: Trata-se de revisão integrativa de literatura, realizada em artigos originais completos publicados através da Biblioteca Virtual de Saúde nas bases de dados: SciELO, LILACS e MEDLINE, em língua portuguesa, inglesa e espanhola, publicados entre 2014 e 2019. Resultados: Foram encontrados 582 artigos nas bases de dados escolhidas, sendo 527 na MEDLINE, 51 na LILACS e 4 na SciELO. Após aplicação dos critérios de elegibilidade do estudo, foram definidos 11 artigos que convergem em grande parte para um evento que necessita de maior atenção dos profissionais e da academia sobre o contexto de sua origem e suas consequências que impactam nas condições de trabalho e refletem na vida extralaboral. Considerações finais: Embora a Síndrome de Burnout seja uma síndrome multifatorial, muitos elementos de sua gênese estão relacionados a sobrecarga de trabalho, ao reduzido reconhecimento profissional, a autoexigência e a outros fatores socialmente induzidos. Tais elementos de gênese individual ou coletiva em muitos casos antecedem o início da atividade profissional e carecem de medidas profiláticas e terapêuticas como também de mais estudos, dimensionando melhor este crescente e complexo problema.

Palavras-Chave: Burnout, Esgotamento profissional, Odontologia.

\section{ABSTRACT}

Objective: To review the literature on the main contributions about Burnout syndrome related to dental surgeons. Methods: This is an integrative literature review, carried out on complete original articles published through the Virtual Health Library in the databases: SciELO, LILACS and MEDLINE, in Portuguese, English and Spanish, published between 2014 and 2019. Results: 582 articles were found in the chosen databases, 527 in MEDLINE, 51 in LILACS and 4 in SciELO. After applying the study's eligibility criteria, 11 articles were defined that largely converge to an event that needs more attention from professionals and academia on the context of its origin and its consequences that impact on working conditions and reflect on life extra-labor. Final considerations: Although Burnout syndrome is a multifactorial syndrome, many elements of its genesis are related to work overload, reduced professional recognition, self-demand, and other socially induced factors. Such elements of individual or collective genesis in many cases precede the beginning of professional activity and lack prophylactic and therapeutic measures as well as more studies, better dimensioning this growing and complex problem.

Key words: Burnout, Emotional exhaustion, Dentistry.

\section{RESUMEN}

Objetivo: Revisar la literatura sobre las principales contribuciones sobre el síndrome de Burnout relacionadas con los cirujanos dentales. Métodos: Esta es una revisión integral de la literatura, realizada en artículos originales completos publicados a través de la Biblioteca Virtual en Salud en las bases de datos: SciELO, LILACS y MEDLINE, en portugués, inglés y español, publicados entre 2014 y 2019. Resultados: Se

\footnotetext{
${ }^{1}$ Centro Universitário do Pará (CESUPA), Belém - PA. * E-mail: wellisson.luigi@hotmail.com

2Universidade do Estado do Pará (UEPA), Belém - PA.

${ }^{3}$ Faculdade Gamaliel (FATEFIG), Tucuruí - PA.

4Universidade Federal do Estado do Pará (UFPA), Belém - PA.
} 
encontraron 582 artículos en las bases de datos elegidas, 527 en MEDLINE, 51 en LILACS y 4 en SciELO. Después de aplicar los criterios de elegibilidad del estudio, se definieron 11 artículos que convergen en gran medida a un evento que necesita más atención de profesionales y académicos sobre el contexto de su origen y sus consecuencias que impactan en las condiciones de trabajo y reflejan en la vida. trabajo extra. Consideraciones finales: Aunque el síndrome de Burnout es un síndrome multifactorial, muchos elementos de su génesis están relacionados con la sobrecarga de trabajo, el reconocimiento profesional reducido, la autoexigencia y otros factores socialmente inducidos. Estos elementos de génesis individual o colectiva en muchos casos preceden a inicio de la actividad profesional y falta de medidas profilácticas y terapéuticas, así como más estudios, dimensionando mejor este problema creciente y complejo.

Palabras clave: Burnout, Agotamiento profesional, Odontología.

\section{INTRODUÇÃO}

Síndrome de Burnout (SB) ou Síndrome do Esgotamento Profissional é definida por uma reação à tensão emocional crônica, tendo como algumas de suas características sentimentos de inutilidade, esgotamento, ansiedade e descontentamento. Quando não há eficácia no método de enfrentamento, ocorrem consequências negativas na área profissional e pessoal (ALMEIDA LA, et al., 2016).

Esta síndrome é constituída por três dimensões: a exaustão emocional, caracterizada por falta de energia e entusiasmo; descrença/despersonalização, a qual refere-se ao desenvolvimento de insensibilidade emocional e; dificuldades no que se refere a eficácia/realização profissional, ou seja, o indivíduo quase sempre se auto-avalia de forma negativa (ZUCOLOTO ML, et al., 2014; CAMPOS JA, et al., 2012).

O estresse, por sua vez, é compreendido como um conjunto de reações que culminam com quebra da homeostasia orgânica decorrente de agressões de ordem física, psíquica, infecciosa e outras. $O$ estresse ocupacional pode ser definido como um processo relacional entre os aspectos cognitivos, emocional e motivacionais do indivíduo com o contexto organizacional (SOUZA AS, et al., 2017; ESTEVES GGL, et al., 2019).

A Síndrome de Burnout é situada no contexto da experiência individual de estresse das pessoas com seu trabalho, ou seja, no contexto mais amplo da organização de sua atividade laboral com consequências sobre as esferas pessoal e social que repercutem na queda da qualidade do trabalho e na saúde física e psicológica do indivíduo. O impacto sobre a qualidade de vida do indivíduo é importante e isto pode ser razão de impacto na qualidade de sua assistência aos pacientes, às suas atividades de gerenciamento de serviços, representando, em muitos casos, aumento de custos relacionados ao serviço de saúde (MASLACH C e LEITER MP, 2016).

O profissional da odontologia está frequentemente exposto a fatores estressores no ambiente de trabalho e fora dele. Nem sempre, entretanto, este profissional possui as habilidades de enfretamento necessárias para dirimir as consequências da exposição a estes fatores. Esta habilidade de enfrentamento denominada por vários autores como coping (CARLOTTO MS, MELO LP, 2016) é o grande desafio desse profissional que tem no ambiente restrito de seu trabalho, parte das razões desta síndrome, pois a tensão da vida moderna, da concorrência, das metas salariais necessárias/desejadas, violência, problemas de ordem familiar, crises e incertezas no cenário econômico no país, etc. um conjunto de fatores a se somar aos eventos específicos de seu ambiente de trabalho.

Os fatores estressores no trabalho têm natureza e intensidade bastante diversificados. É importante que no contexto organizacional do trabalho, esses fatores sejam adequadamente identificados e que as atividades que possam minorá-los sejam implementadas. Grande parte das medidas gerenciais que podem criar essas possibilidades e gerar mudanças diante dos fatores estressores, é negligenciada pela gestão ou pelo próprio profissional, o que gera dificuldades no gerenciamento de tensões e perda de qualidade do trabalho do indivíduo e da equipe (RODRIGUEZ SYS, CARLOTTO MS, 2014).

Apesar de esta síndrome ser ainda pouco explorada na literatura da odontologia no Brasil, o cirurgiãodentista tem sido apontado como um dos profissionais vulneráveis a diversos riscos a ela relacionados. A 
profissão de cirurgião-dentista tem sido apontada como uma das que se expõe a riscos ocupacionais peculiares da sua atuação, como a demanda de precisão, concentração e responsabilidade nos procedimentos, elevada carga horária de trabalho, postura de trabalho de risco ergonômico, necessidade de cuidado e atenção no manejo de elementos químicos e biológicos e atividades de administração e organização do ambiente de trabalho (SOUZA AS, et al., 2017; CAMPOS JA, et al., 2012; ZUCOLOTO ML, et al., 2014).

Percebe-se hoje, uma grande necessidade de produção de conhecimento sobre a SB na área da odontologia. Os estudos, em grande parte, estão direcionados a estudantes de graduação que, embora relevantes, precisam ser ampliados aos profissionais em dimensão mais expressiva. Há uma profícua produção de investigação sobre Síndrome de Burnout em docentes no Brasil, mas esta produção está muito concentrada em instituições públicas de ensino (DIEHL L, CARLOTTO MS, 2015). Há necessidade de dimensioná-la adequadamente em contextos específicos para, em seguida, trabalhar as intervenções de prevenção e controle.

Parte desses estudos avaliam o cirurgião dentista que também exerce a atividade docente paralelamente. Ao somar essas duas atividades profissionais, um incremento de risco ou de proteção poderá ser adicionado às condições laborais que afetam a saúde mental do cirurgião dentista. Serão fatores de proteção quando esta segunda atividade representa uma quebra da rotina estressora da assistência, da pressão das metas de procedimentos e atendimento de número de pacientes, e quando esta segunda atividade representa em grande parte uma atividade prazerosa.

Por outro lado, alguns autores destacam que o docente vem perdendo autonomia sobre suas tarefas, trabalha sob forte tensão emocional, nem sempre tem reconhecimento financeiro em sua atividade profissional e a ascensão na carreira não é uma regra geral sobretudo nas instituições privadas onde a exigência e a possibilidade de perda de emprego são maiores. A necessidade de atualização constante e a pressão às adaptações a novas tecnologias metodológicas e metas de aprendizado concorrem para uma pressão que podem ser extenuantes a este profissional (TYTHERLEIGH MY, et al., 2005).

Diversos estudos argumentam que o burnout surgiu como um problema social, sendo considerado como uma síndrome psicológica, oriunda de uma tensão emocional constante, vivida por profissionais que necessitam conviver constantemente com pessoas que precisam de sua assistência em saúde. Desta forma, é possível se afirmar que, a partir de uma perspectiva psicossocial, a síndrome de burnout é entendida enquanto um processo, no qual as relações de trabalho e interpessoais contribuem positivamente ou negativamente para o seu desenvolvimento (SOUSA SAS, et al., 2017).

A síndrome de burnout como resultado do esgotamento profissional, tendo como consequência um déficit na qualidade dos serviços prestados, além do sofrimento pessoal, exaustão física, insônia, aumento do uso de álcool e drogas e ainda problemas familiares, provoca o desenvolvimento de atitudes negativas, assim como uma auto avaliação negativa. Profissionais estes que podem estar sendo expostos a esses fatores estressores desde o período de formação acadêmica e se estendendo por toda sua vida profissional, visto que a relação com o paciente se configura como um intenso fator estressor (OLIVEIRA PHB, OLIVEIRA AHA, 2018).

Estudos mostram que esse desequilíbrio na saúde do trabalhador pode acarretar o aumento nos índices de absenteísmo, gerando licenças médicas, sendo vistas negativamente pelo empregador, e gerando consequentemente a necessidade por parte da organização, de reposição de funcionários, transferências, novas contratações, novos treinamentos, entre outras despesas. Afetando diretamente nos serviços, seja em relação à produtividade ou à lucratividade da empresa, do órgão ou da instituição pública ou privada (ANDRADE PS, et al., 2012).

Esta síndrome atinge em diferentes graus, diversas categorias profissionais, diferentes instituições e pessoas necessitam ter um olhar mais atento por parte da academia, pois representa razão de sofrimento importante no ambiente do trabalho, repercutindo fora de seus limites. As mudanças que ocorrem no cenário laboral e fora dele, podem repercutir negativamente na vida do profissional, induzindo a este sofrimento cuja percepção pode ser precoce ou tardia. Refletir sobre esse problema à luz da literatura produzida nos últimos 
anos, poderá contribuir com uma percepção melhor do problema. O presente trabalho tem como objetivo realizar uma revisão integrativa de literatura sobre o tema Síndrome de Burnout em cirurgiões-dentistas, uma categoria cujos problemas relacionados a saúde mental decorrentes do trabalho precisam ser adequadamente dimensionados em sus causas, consequências e formas de intervenção.

Nesta revisão de literatura não houve coleta de dados realizada em seres humanos ou acesso a qualquer tipo de informação confidencial a eles relacionados. Portanto, não requer aprovação por um comitê de ética.

\section{MÉTODOS}

Trata-se de revisão integrativa de literatura, obedecendo as normas pertinentes de levantamento, seleção, ordenação e síntese de material publicado por outros autores. Para este fim foram seguidas as seguintes etapas: definição do tema e seleção da questão de pesquisa; Definição dos critérios de inclusão e exclusão; Pré-seleção, seguido de seleção dos estudos; Estabelecidos das categorias dos estudos já selecionados; Análise e interpretação dos resultados; Apresentação (síntese) da revisão (CELESTINO JÚNIOR, et al., 2018).

O tema definido na pesquisa foi estresse e Síndrome de Burnout em profissionais da odontologia. A questão de pesquisa definida foi: Quais os principais achados da literatura relacionados ao estresse e síndrome de burnout na odontologia? Quais as principais situações que favorecem o surgimento desses fenômenos?

Figura 1 - Critério de inclusão e exclusão.

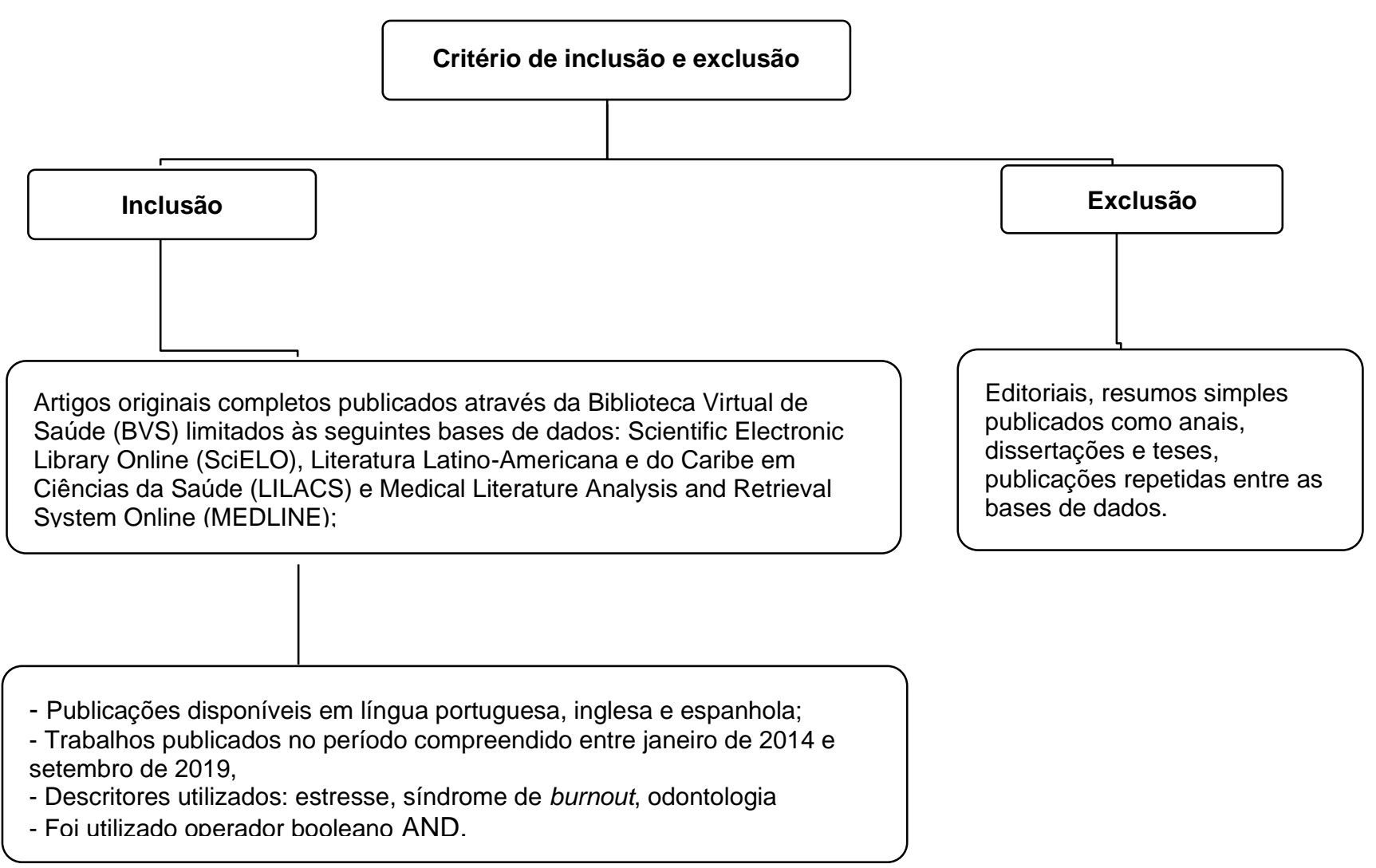

Fonte: Pinheiro WLL, et al., 2020.

\section{RESULTADOS}

Depois de aplicar os descritores supramencionados foram encontrados 582 artigos nas bases de dados escolhidas, sendo 527 na MEDLINE, 51 na LILACS e 4 na SciELO. Após leitura do título, resumo e em seguida o texto completo, utilizando os critérios de elegibilidade do estudo, foram definidos 11 estudos sendo 5 na base de dados MEDLINE, 5 na LILACS e 1 na SciELO. 


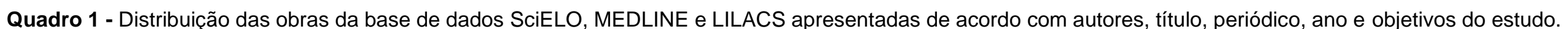

\begin{tabular}{|c|c|c|c|c|}
\hline Autores e ano & Título & Revista & Objetivo do estudo & Base de dados \\
\hline \multirow[t]{2}{*}{ MAGRI LV, et al., (2016) } & $\begin{array}{l}\text { Relationship between } \\
\text { temporomandibular disorder } \\
\text { symptoms sings and Burnout } \\
\text { syndrome among dentistry student. }\end{array}$ & \multirow[t]{2}{*}{ Rev Dor. São Paulo } & \multirow{2}{*}{$\begin{array}{l}\text { Analisar de forma prospectiva as dimensões } \\
\text { da síndrome de Burnout, e sua relação com a } \\
\text { percepção dos sinais e sintomas de disfunção } \\
\text { temporomandibular em uma mostra de } \\
\text { estudantes de graduação em odontologia. }\end{array}$} & \multirow[t]{2}{*}{ SciELO } \\
\hline & $\begin{array}{l}\text { Relação entre os sintomas da } \\
\text { disfunção temporomandibular e a } \\
\text { síndrome de Burnout em estudantes } \\
\text { de odontologia. }\end{array}$ & & & \\
\hline \multirow[t]{2}{*}{ HAKANEN J, et al., (2014) } & $\begin{array}{l}\text { Crossover of Exhaustion between } \\
\text { dentists and dental nurses. }\end{array}$ & \multirow[t]{2}{*}{$\begin{array}{l}\text { Wiley Online } \\
\text { Library }\end{array}$} & \multirow{2}{*}{$\begin{array}{l}\text { O objetivo desse estudo foi investigar as } \\
\text { condições sob as quais a exaustão } \\
\text { relacionada ao trabalho pode transmitir de } \\
\text { dentistas para auxiliares e vice-versa. }\end{array}$} & \multirow[t]{2}{*}{ MEDLINE } \\
\hline & $\begin{array}{l}\text { Cruzamento da exaustão entre } \\
\text { dentistas e enfermeiros. }\end{array}$ & & & \\
\hline \multirow[t]{2}{*}{ MAFLA AC, et. al., (2015) } & $\begin{array}{l}\text { Burnout prevalence and correlates } \\
\text { amongst Colobian dental students: the } \\
\text { STRESSCOSE study. }\end{array}$ & \multirow[t]{2}{*}{$\begin{array}{l}\text { European Journal } \\
\text { of Dental Education }\end{array}$} & \multirow{2}{*}{$\begin{array}{l}\text { Determinar a prevalência de burnout uma } \\
\text { considerável amostra de estudes de } \\
\text { graduação em odontologia da Colômbia e } \\
\text { investigar suas relações psicossociais e } \\
\text { educacionais. }\end{array}$} & \multirow[t]{2}{*}{ MEDLINE } \\
\hline & $\begin{array}{l}\text { Prevalência de burnout e correlatos } \\
\text { entre estudantes colombianos de } \\
\text { odontologia: o estudo STRESS CASE }\end{array}$ & & & \\
\hline \multirow[t]{2}{*}{ MACHE S, et al., (2015) } & $\begin{array}{l}\text { Prevention of study-related stress } \\
\text { symptoms: health-promoting behavior } \\
\text { among dental students. }\end{array}$ & \multirow[t]{2}{*}{$\begin{array}{l}\text { Wien Med } \\
\text { Woxhenschr }\end{array}$} & \multirow[t]{2}{*}{$\begin{array}{l}\text { Avaliar a prevalência de sintomas de burnout } \\
\text { e atividades de prevenção contra em } \\
\text { estudantes de odontologia. }\end{array}$} & \multirow[t]{2}{*}{ MEDLINE } \\
\hline & $\begin{array}{l}\text { Prevenção de sintomas de estresse } \\
\text { relacionados ao estudo: } \\
\text { comportamento promotor da saúde } \\
\text { entre estudantes de odontologia. }\end{array}$ & & & \\
\hline \multirow[t]{2}{*}{ SINGH P, et al., (2016) } & $\begin{array}{l}\text { Systematic review: factors contributing } \\
\text { to burnoutin dentistry }\end{array}$ & \multirow[t]{2}{*}{$\begin{array}{l}\text { Ocupational } \\
\text { Medicine }\end{array}$} & \multirow{2}{*}{$\begin{array}{l}\text { Identificar os fatores mais significantes } \\
\text { associados com burnout em dentistas e } \\
\text { estudantes de odontologia publicados na } \\
\text { literatura. }\end{array}$} & \multirow[t]{2}{*}{ MEDLINE } \\
\hline & $\begin{array}{l}\text { Revisão sistemática: fatores que } \\
\text { contribuem para o burnout em } \\
\text { odontologia }\end{array}$ & & & \\
\hline
\end{tabular}

REAS/EJCH | Vol.Sup.n.51 | e3270 | DOI: https://doi.org/10.25248/reas.e3270.2020 Página 5 de 10 


\begin{tabular}{|c|c|c|c|c|}
\hline Autores e ano & Título & Revista & Objetivo do estudo & Base de dados \\
\hline \multirow[t]{2}{*}{ DEEB GR, et al., (2017) } & $\begin{array}{l}\text { Depression and suicidal ideation in } \\
\text { dental and dental hygiene students }\end{array}$ & \multirow[t]{2}{*}{ Eur J Dent } & \multirow{2}{*}{$\begin{array}{l}\text { O objetivo desse estudo foi avaliar a relação } \\
\text { burnout, sintomas de depressão e ideação } \\
\text { suicida em estudantes de odontologia e } \\
\text { estudantes de auxiliar odontológico e avaliar o } \\
\text { gênero, tipo de planejamento e ano de estudo. }\end{array}$} & \multirow[t]{2}{*}{ MEDLINE } \\
\hline & $\begin{array}{l}\text { Depressão e ideia suicida em } \\
\text { estudantes de odontologia e higiene } \\
\text { dental }\end{array}$ & & & \\
\hline \multirow[t]{2}{*}{ PEREZ F, et al., (2016) } & $\begin{array}{l}\text { Association of burnout with stress, } \\
\text { coping strategies, and vocational } \\
\text { satisfaction in Chilean clinical } \\
\text { students. }\end{array}$ & \multirow[t]{2}{*}{ J Oral Res } & \multirow[t]{2}{*}{$\begin{array}{l}\text { O objetivo desse estudo foi analisar o efeito do } \\
\text { estresse percebido, estratégias de tratamento } \\
\text { e satisfação profissional relacionado a } \\
\text { severidade do burnout em estudantes chilenos } \\
\text { de odontologia durante os anos de clínica. }\end{array}$} & \multirow[t]{2}{*}{ LILACS } \\
\hline & $\begin{array}{l}\text { Associação de burnout com estresse, } \\
\text { estratégias de enfrentamento e } \\
\text { satisfação profissional em estudantes } \\
\text { clínicos chilenos. }\end{array}$ & & & \\
\hline $\begin{array}{l}\text { NEVES CPD, RIBEIRO } \\
\text { DM, (2016) }\end{array}$ & $\begin{array}{l}\text { Burnout em estudantes de graduação } \\
\text { em Odontologia }\end{array}$ & Revista da ABENO & $\begin{array}{l}\text { Objetivou verificar a prevalência da Síndrome } \\
\text { de Burnout entre estudantes de graduação de } \\
\text { odontologia de } 1^{\underline{a}}, 5^{\underline{a}} \text { e } 10^{\underline{a}} \text { fases da UFSC. }\end{array}$ & LILACS \\
\hline $\begin{array}{l}\text { CAMPOS FM, et al., } \\
(2017)\end{array}$ & $\begin{array}{l}\text { Antecedentes académicos como } \\
\text { predictores de Burnout em } \\
\text { estudiantes de primer año de } \\
\text { Odontologia }\end{array}$ & $\begin{array}{l}\text { Educación Médica } \\
\text { Superior }\end{array}$ & $\begin{array}{l}\text { Associar os ambientes acadêmicos dos } \\
\text { estudantes de primeiro ano de odontologia e } \\
\text { seus níveis de Burnout. }\end{array}$ & LILACS \\
\hline CRUZ AD, et al., (2017) & $\begin{array}{l}\text { Condições de saúde oral e indicativos } \\
\text { de síndrome de Burnout em } \\
\text { estudantes de Odontologia } \\
\text { sedentários e atletas }\end{array}$ & Revista da ABENO & $\begin{array}{l}\text { Comparar condições de saúde oral e os } \\
\text { indicativos de síndrome de burnout em } \\
\text { estudantes de Odontologia sedentários e } \\
\text { atletas. }\end{array}$ & LILACS \\
\hline \multirow[t]{2}{*}{$\begin{array}{l}\text { NASCIMENTO VL, et al., } \\
(2018)\end{array}$} & $\begin{array}{l}\text { Burnout Syndorme among dental } \\
\text { professor; a cross-sectional study }\end{array}$ & \multirow[t]{2}{*}{ Revista da ABENO } & \multirow{2}{*}{$\begin{array}{l}\text { Avaliar a prevalência da síndrome de burnout } \\
\text { entre professores de universidades de } \\
\text { odontologia brasileiras e investigar quais } \\
\text { fatores sócio demográficos estão associados } \\
\text { com esta condição. }\end{array}$} & \multirow[t]{2}{*}{ LILACS } \\
\hline & $\begin{array}{l}\text { Síndrome de Burnout entre professor } \\
\text { de Odontologia; um estudo } \\
\text { transversal }\end{array}$ & & & \\
\hline
\end{tabular}

Fonte: Pinheiro WLL, et al., 2020. 


\section{DISCUSSÃO}

Singh P, et al., (2016) realizando estudo de revisão, identificou vários fatores importantes que contribuem para a SB, mas destacam que esta síndrome é multifatorial e que pode se desenvolver no início da profissão. Dentre os fatores relacionados verificaram: idade mais jovem, sexo masculino, sobrecarga de trabalho, horário de trabalho e certos tipos de personalidade. Estes autores destacam também que a maior parte da literatura mostra que a síndrome é mais comum em mulheres, apesar de alguns estudos mostrarem que é maior em homens.

Além disso, verificou-se que mais de $26 \%$ da equipe odontológica estava em risco severo em adquirir a síndrome. Quanto mais prática clínica os profissionais e os estudantes tiverem, as maiores chances de estresse e esgotamento profissional e, portanto, maior possibilidade de adquirir a SB. Em relação aos alunos de residências, mestrado e doutorado, verifica-se que os últimos anos de estudo são os mais complexos. A diferença no estilo de vida também é um ponto a ser destacado, já que gravidez, pressão familiar, entre outras situações de convívio social podem acabar agravando a Síndrome de Burnout e se não for tratada corretamente, as chances desses indivíduos adquirirem depressão e suicídio aumentam consideravelmente.(SINGH P, et al.,2016).

Avaliando a SB entre professores de curso de graduação em odontologia, Nascimento VL, et al., (2018) verificaram no grupo por eles estudados que as principais dimensões da síndrome estavam relacionadas aos fenômenos de ordem pessoal e síndrome relacionada ao ambiente de trabalho. Apontam ainda para a necessidade de realização de pesquisas que venham dar maior visibilidade e consistência para este fenômeno.

Ao avaliarem a relação entre SB, sintomas depressivos e ideação suicida em dentistas e estudantes de odontologia, Deeb GR, et al., (2017) verificaram que $40 \%$ dos dentistas e $38 \%$ estudantes se enquadravam nos critérios de Burnout. 9\% dos dentistas e $9 \%$ dos estudantes apresentavam quadros compatíveis com depressão moderada. Em relação a ideação suicida, este estudo encontrou $6 \%$ dos dentistas com sintomatologia compatível e entre os estudantes esse percentual foi de $9 \%$.

Embora o foco principal do estudo em tela esteja voltado para o cirurgião dentista já em atuação na profissão, há achados relacionados a exposição a fatores de risco ou condição pessoal que antecedem mesmo a imersão no universo profissional. É o que aponta o estudo de Magri LV, et al., (2016) ao avaliarem um grupo de estudantes de odontologia em três períodos diferentes de sua graduação (primeiro, segundo e terceiro ano). Esses autores avaliaram a correlação entre disfunção da articulação temporomandibular e a síndrome de Burnout e verificaram que no público por eles avaliados, havia correlação entre a exaustão emocional e a percepção de sinais e sintomas desta patologia articular notadamente em estudantes do sexo feminino com tendência de aumento com a progressão da graduação.

É importante salientar que a Síndrome de Burnout traz consequências não somente para para a saúde mental do profissional, como por exemplo alterações orgânicas nos níveis do hormônio Cortisol que de maneira indireta pdoerá refletir na qualidade de execução do trabalho. Alguns autores (NEVES CPD, RIBEIRO DM, 2016) destacam em seu estudo que aproximadamente $65 \%$ dos participantes da pesquisa dormem apenas entre 6 e 8 horas e $35 \%$ dormem menos de 6 horas diárias. Isto poderá interferir diretamente na qualidade de vida e desempenho profissional.

Em estudo realizado com 5600 estudantes de odontologia de uma universidade de Bogotá, Mafla AC, et al., (2015) encontraram 7\% de estudantes que apresentaram SB. Ao aprofundarem sua investigação, verificaram que elementos do ambiente pessoal e educacional estavam associadas à síndrome de burnout. No conjunto de elementos que compõem causas associadas a síndrome, a carga de trabalho e a autoexigência com resultados tiveram influência relevante em relação a síndrome neste grupo.

Por outro lado, no estudo realizado por Neves CP e Ribeiro DM (2016), foi encontrada uma prevalência de $10,89 \%$ de estudantes compatíveis com síndrome de burnout. Concorreram para este percentual, causas como as reduzidas horas semanais de lazer por parte dos estudantes, poucas horas diárias de sono, autopercepção de desempenho acadêmico negativo, percepção de desempenho dos professores ruim, além 
de aspectos relacionados às instalações físicas da faculdade. Em adição, foram também relatados consumo de medicamentos e ideação de desistência do curso.

Estudo realizado com estudantes do ensino médio e estudantes do primeiro ano de odontologia verificouse associação estatisticamente significante entre os casos da síndrome de burnout entre esses dois grupos significando que mesmo antes de adentrar ao curso de graduação, há estudantes com antecedentes da síndrome (CAMPOS FM, et al., 2017).

É de grande relevância o que destaca o estudo de Hakanen J, et al., (2014) ao apontar a influência que pode ter o comportamento de um profissional sobre os outros membros da equipe. Eles avaliaram que profissionais com função de liderança na equipe (dentista) sobre os demais (auxiliares como ASB ou TSB) exercem forte influência sobre estes, tanto positiva quanto negativamente, isto é, situações de esgotamento psicológico podem passar de dentistas esgotados para seus auxiliares. De igual forma, as condições favoráveis de colaboração e harmonia frequentes foram consideradas fatores de proteção associados a sinais/sintomas de esgotamento no trabalho. Concluem estes autores que sintomas compatíveis com síndrome de burnout tem elementos de origem individual, mas também podem ser socialmente induzidos.

A prevenção ou alívio do quadro sindrômico de burnout pode ser iniciada ainda na vida acadêmica, quando já podem ser identificados sintomas ou condições de risco que sinalizam a síndrome. Embora o percentual de pessoas em condição de risco ou já com a síndrome varie entre os diversos estudos, é uma condição cujo diagnóstico deve ser realizado o mais precoce possível, pois as condições de risco de morbidade psicológica nem sempre são claros. Além da identificação do problema, há necessidade de se atuar no sentido de atenção aos indivíduos que se encontram nessa condição como também reduzir as condições de estresse identificáveis no ambiente acadêmico (MAFLA AC, et al., 2015). Em grande medida, a reflexão sobre o problema, a identificação e suas intervenções eficazes reduzirão as chances de se repetirem na vida profissional.

A formação de um cirurgião antecipa muito das experiências de um profissional. De fato, há diferentes situações de formação durante o curso que representam fatores de risco psicológico. Apesar disso, há poucos estudos apontando formas de intervenção para melhor enfrentamento das situações que são razões de esgotamento mental. Em estudo realizado na Alemanha com 239 estudantes de odontologia, verificou-se que $38 \%$ deles presentavam sintomas de exaustão emocional. Esse conjunto de estudantes relatou que para minimizar esta situação, desenvolviam atividades como exercícios físicos e, por vezes, uso de narcóticos (MACHE S, et al., 2015).

Segundo Cruz AD, et al., (2017), estudantes considerados sedentários são mais sensíveis a tensões oriundas no ambiente acadêmico. Tal estudo converge às evidências levantadas em outros estudos que destacam as atividades físicas como fator de proteção ao desenvolvimento de síndrome de Burnout.

Mache S, et al., (2015) destacam em seu estudo que há grande necessidade de implementar ações promoção da saúde voltadas para as pessoas que se encontram expostas ao risco de desenvolverem esgotamento psicológico.

Esses achados apontam para a necessidade de introduzir medidas preventivas durante a formação dos estudantes. Estudos como o de Deeg GR, et al., (2017) apontam para uma variedade de atividades viáveis na promoção de saúde que auxiliam na diminuição do estresse, como por exemplo: ioga, alimentação saudável, esportes, atividades de relaxamento e redução de alguns hábitos desfavoráveis como o uso de álcool ,drogas tranquilizantes e tabagismo. Entretanto, estes ainda são bastantes consumidos pelos estudantes no intuito de lidar com sofrimento e problemas psicológicos. Neste estudo realizado na Alemanha mostrou que os homens fazem mais uso de drogas e álcool, enquanto as mulheres fazem atividades relaxantes como cozinhar e se alimentar de forma saudável.

Além dos problemas relacionados a articulação temporomandibular, outros fatores físicos podem se desencadear, apesar de não elucidados pelo autor tem relação direta com o estresse como lesões de carie e doença periodontal, causa esta já bem estudada pela periodontia (CRUZ AD, et al., 2017). Neste mesmo estudo o autor cita algumas variáveis de origem bucal em pacientes sedentários como sangramento a 
sondagem, profundidade de sondagem e achados radiográficos, fato esse que reforça a importância do psicológico na homeostase geral.

Outro tipo de profissional bastante acometido pela SB é o dentista que também é professor universitário pois no artigo de Nascimento VL, et al. (2018) é citado a preocupação diária com múltiplas responsabilidades materiais e equipamentos disponíveis, além disso o professor precisa estar atento em diversos lugares como a pratica clínica, sala de aula , laboratórios e no meio administrativo também ,ademais sempre se mantendo atualizado quanto à prática acadêmica. Dessa forma, o estresse e excesso de reponsabilidade podem ser um gatilho para o desenvolvimento desta síndrome.

O estudo de Cruz AD, et al., (2017) comprovou que existe uma correlação positiva fraca entre o sangramento a sondagem com a exaustão emocional, que seria o primeiro indicativo da síndrome, ainda afirmaram que os estudantes que praticavam esportes possuíam melhores condições de enfrentamento de problemas emocionais

Em estudo realizado com 244 estudantes chilenos Péres F, et al., (2016) verificaram falhas no enfrentamento das situações que significavam altos níveis de estresse e baixos níveis de satisfação profissional presente e futura. Tais falhas relacionavam a não identificação de indivíduos com o problema e limitação das ações aos que já as apresentavam. Este estudo também verificou que a SB é uma síndrome de exaustão emocional, predominantemente dos profissionais de saúde e que os dentistas são os que possuem a maior prevalência dessa síndrome, já que é uma a profissão fisicamente e psicologicamente exigente.

As consequências do burnout incluem baixo desempenho no trabalho, associado à rejeição e vontade de abandonar a profissão, além de sentimento de fracasso e baixa autoestima e que, quanto maior a demanda de pacientes, maiores os níveis de insegurança. A prevalência de burnout em estudantes chilenos é maior do que em outros países, sendo as mulheres e os alunos do $4^{\circ}$ período os mais afetados (PÉRES F, et al., 2016).

\section{CONSIDERAÇÕES FINAIS}

Os estudos selecionados mostraram a presença de diversos fatores relacionados ao estresse e a síndrome de burnout em cirurgiões-dentistas, tais como fadiga, baixos níveis de apoio social, entre outros. Estes fatores já estão presentes ainda na graduação e ainda há um grupo de risco mais propenso e sensível ao estresse ocupacional: as mulheres, os sedentários e os profissionais de serviço público. Desta forma, programas de prevenção e combate à síndrome de burnout, além de exercícios de alongamento, mostram-se eficazes para amenizar as consequências da alta carga de trabalho. Estas alternativas, além de eficazes, são muito práticas. Por fim, verifica-se que novos estudos devem ser conduzidos, principalmente qualitativos, com intuito de ampliar e diversificar a coleta de dados, já que existem poucos estudos no Brasil e em português, o que poderá contribuir com a melhoraria das condições de trabalho dos cirurgiões-dentistas e sua saúde mental.

\section{REFERÊNCIAS}

1. ANDRADE PS, et al. Prazer e Dor na Docência: revisão bibliográfica sobre a Síndrome de Burnout. Saúde Soc. São Paulo, 2012; 21(1): 129-140.

2. CAMPOS FM, et al. Antecedentes académicos como predictores de Burnout en estudiantes de primer año de Odontología. Educación Médica Superior, 2017; 31(2).

3. CARLOTTO MS, CÂMARA SG. Riscos psicossociais associados à síndrome de burnout em professores universitários. Avances en Psicología Latinoamericana, 2017; 55(3): 447-457.

4. CARLOTTO MS, MELO LP. Prevalência e preditores de Burnout em Bombeiros. Psicologia ciência e profissão, 2016; 36(3).

5. CRUZ AD, et al. Condição de saúde oral e indicativos da síndrome de Burnout em estudantes de Odontologia sedentários e atletas. Revista da ABENO, 2017; 17(2): 97-106.

6. DEEB GR, et al. Burnout, depression and suicidal ideation in dental and dental hygiene students. Eur J Dent Educ, 2018; 22: $70-74$.

7. DIEHL L, CARLOTTO MS. Síndrome de Burnout: indicadores para a construção de um diagnóstico. Psicologia

8. Clínica, 2015; 27(2): 161-179.

9. HAKANEN JJ, et al. Crossover of Exhaustion between Dentists and Dental Nurses. Stress Health, 2014; 30: 110-121.

10. MACHE S, et al. Prevention of study-related stress symptoms: health-promoting behavior among dental students. Wien Med Wochenschr, 2015; 165: 100-106. 
11. MAFLA AC, et al. Burnout prevalence and correlates amongst Colombian dental students: the STRESSCODE study. Eur J Dent Educ, 2015; 19(4): 242-250.

12. MAGRI LV, et al. Relationship between temporomandibular disorder symptoms signs and Burnout syndrome among dentistry students. Rev Dor. São Paulo, 2016; 17(3): 171-7.

13. MASLACH C, LEITER MP. Stress: Concepts, cognition, emotion, and behavior. Handbook of Stress Series, 2016; 1: 351-357.

14. NASCIMENTO VL, et al. Burnout Syndrome among Dental professors: a cross-sectional study. Revista da ABENO, 2018; 18(2): 62-71.

15. NEVES CP, RIBEIRO DM. Burnout em estudantes de graduação em odontologia. Revista da ABENO, 2016; 16(1): 3949.

16. OLIVEIRA PHB, OLIVEIRA AHA. Síndrome de Burnout em acadêmicos de Odontologia. J Dent Pub H, Salvador, 2018; 9(4): 263-269.

17. PÉREZ F, et al. Association of burnout with stress, coping strategies and vocational satisfaction in Chilean clinical dental students. J Oral Res, 2016; 5(8): 320-327.

18. RODRIGUEZ SYS, CARLOTTO MS. Prevalência e Fatores Associados à Síndrome de Burnout em Psicólogos. Ciência e Trabalho, 2014; 16(51): 170-176.

19. SINGH P, et al. Systematic review: factors contributing to burnout in dentistry. Occupational Medicine, 2016; 66: $27-31$.

20. SOUZA SAS, et al. Síndrome de Burnout: o Cirurgião-Dentista docente e o esgotamento laboral crônico. Revista Campo do Saber, 2017; 3(2).

21. TYTHERLEIGH MY, et al. Occupational stress in UK higher education institutions: a comparative study of all staff categories. Higher Education Research and Development, 2005; 24(1): 41-61. 